\title{
3. Contemporary Concepts of Time in Western Science and Philosophy
}

\author{
Peter J. Riggs
}

Time present and time past

Are both perhaps present in time future

And time future contained in time past.

If all time is eternally present

All time is unredeemable.

TS Eliot

\section{Introduction}

The term 'deep time' denotes vast, extremely remote periods of (natural or other) history - distant and extensive spans of time that are almost beyond the grasp of the human mind. In western science, deep time is used to refer to eras dating back to the formation of the Earth (about 4.5 billion years ago) as indicated by empirical evidence, for example, the geological record. The geologist Stephen J Gould provides the following portrayal of deep time in his book Time's Arrow, Time's Cycle:

[I]mposed by geology ... 'deep time' ... [is] the notion of an almost incomprehensible immensity ... so outside our ordinary experience [and] so alien that we can really only comprehend it as metaphor. ${ }^{1}$

Disciplines other than geology have also embraced notions of deep time, as have cross-disciplinary studies. ${ }^{2}$ Insights from cross-cultural views of time (especially Australian Indigenous perspectives) are not discussed here but are dealt with in several other chapters.

What might be thought of time itself? All conscious human beings seem to perceive time. Time provides us with the order in which events occur (their temporal order) and the order of our perceptions of the world around us. The perplexing nature of time has been more contemplated, speculated, written

\footnotetext{
Gould 1987: 2-3.

2 See, for example: Douglas 2010; Shryock and Smail 2011.
} 
and debated about over the ages than virtually any other subject, with the possible exception of religion. Yet time seems more elusive than the vast majority of other metaphysical concepts. Even with the advancement of modern physics (that is, physics since the beginning of the twentieth century) we only have an elementary understanding of time. The nature of time remains both puzzling and bewildering! An oft-quoted statement by the early Christian philosopher and cleric Saint Augustine (354-430 AD) captures much of this sentiment:

What then, is time? If no one asks me, I know. If I wish to explain it to him who asks, I know it not. ${ }^{3}$

The significance of knowledge about time cannot be overstated, for it goes to the core of human consciousness, perception, communication and of our desire to understand ourselves and the universe which we inhabit. Although we are better off today in respect to knowledge about time than was Saint Augustine, time is still an enigma that western philosophy and physical science have not been able to solve, despite time explicitly appearing in the mathematical expression of the fundamental laws of physics. The scientific perspective of time, however, owes much to modern physics, as observed by physicist Carlo Rovelli:

[T] he development of theoretical physics has modified substantially the «natural» notion of time. ${ }^{4}$

Nevertheless, there is no consensus amongst scientifically literate philosophers or among physicists about the nature of time. Neither is there any consensus on which aspects of time are genuine features of reality. Time remains mysterious, for we lack an understanding of time at a basic physical level.

An increase in our knowledge about time would not only bring a better appreciation of the workings of the universe but also of our place as conscious beings in the cosmos. In this chapter, the principal tenets of those theories of time that have attracted and/or still retain support amongst philosophers and physicists will be summarised together with recent and potential advances. These advances may help to illuminate the deep structure of time. We will begin by discussing concepts of time in theories of modern physics and then proceed to consider time as found in contemporary western analytic philosophy.

3 Quoted in Fraser 1987: 35

4 Rovelli 1995: 84. 


\section{Physical concepts of time}

This section commences by acknowledging that science is an empirically based enterprise which deals only with natural phenomena. The outputs of science are physical theories and laws of nature. These laws may be thought of as general statements about causal connections between events (deterministic laws) or general statements about the probability of events (non-deterministic laws). Physics is considered to be the most mature physical science because of its quantitative methods of gathering evidence and theory development, its ability to make precise measurements, its rigorous empirical testing regime, and its criteria for eliminating theories that have failed to agree with experimental data. Only those theories in physics that meet these stringent requirements remain in contention. Time features significantly in both the conduct of physics and as a characteristic of physical theories themselves.

There are certain aspects of physics that are relevant to the scientific perspective of time. First, we must recognise that physics has shown that the common sense view of time is mistaken. The public at large clings to a notion of time that remains firmly entrenched in everyday experience. This notion requires time to be the same for everyone everywhere, regardless of their location or motion. Time is thus accepted as a physical absolute. GJ Whitrow described this attitude in his influential treatise, The Natural Philosophy of Time:

$[\mathrm{M}]$ ost people still have the feeling that time is something that goes on of its own accord unaffected by anything else ...5

It is known from the Special Theory of Relativity (and supported by numerous experiments to extraordinary accuracy) that time is not the same for different observers. While it seems highly counter-intuitive, there is no such thing as an absolute time.

Second, the fundamental laws of physics do not contain any terms that specify an objective present moment (the 'now') even though human consciousness is only aware of the 'now', not the past nor the future. The present moment is completely absent from the equations of physics! ${ }^{6}$

Third, two orientations of time can be specified in physics that match with conscious experience (especially in western thought). Typically, time is graphically represented in western society by a straight line. This fits with our intuitive sense of time as being serially ordered since a straight line is obviously linear and has two orientations - extending to the left and to the right. The two

5 Whitrow 1980: 59.

6 Denbign 1981: 4; Greene 2004: 131. 
orientations of time, which we call earlier and later, correspond to left and right in the straight line representation of time. ${ }^{7}$ Nonetheless, as explained in the previous chapter, this varies across cultures.

Fourth, the fundamental laws of physics do not distinguish between past and future. ${ }^{8}$ All the equations of fundamental physics can be solved for either of the two orientations of time. In other words, these equations can supply results for physical phenomena that will occur (prediction) and results for physical phenomena that have already occurred (retrodiction). The technical phrase for this is that the form of the fundamental laws of physics is time-reversal invariant.

Fifth, a very obvious fact about the universe and time is the existence of (so-called) 'irreversible' processes. An irreversible process may be defined as a process that alters the state of a physical system such that no other (naturally occurring) process can restore the system to its original state at a later time. The coffee and milk in your cup, for example, always spontaneously mix. We never observe coffee and milk naturally staying separate nor un-mixing spontaneously, despite such events not being excluded by the fundamental laws of physics. ${ }^{9}$ The term 'temporal asymmetry' is used to denote the fact that irreversible processes occur only along one orientation of time (the orientation called later). This need not imply anything about a structural asymmetry of time itself but merely refer to processes occurring in time. ${ }^{10}$ At a human level, temporal asymmetry describes our experiences of having memories of the past and not of the future. We shall see that some philosophical accounts of time rule out the inverses of irreversible processes such as a broken egg reassembling itself spontaneously, regardless of these inverse processes not being forbidden by the fundamental laws of physics.

\section{The operational definition of time}

Time has always been an essential element in the study of astronomy and in navigation. ${ }^{11}$ Aside from time in the Theories of Relativity (see below), science has tended to be pragmatic in respect to time, making use of the purely operational definition - time is that which clocks measure. ${ }^{12}$ In pursuit of better means of testing physical theories, progressively more accurate technologies have been developed for the measurement of time $^{13}$ (to the current stage where time intervals

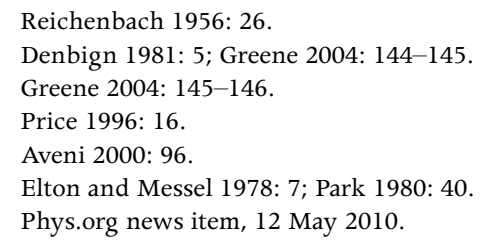


can be determined to an accuracy of 12 attoseconds ${ }^{14}$ ). Although the operational definition of time and higher accuracies of time measurement are essential for practical, observational, and experimental purposes, these operational aspects do not enlighten us about the nature of time. Further, in most of the equations of physics, time is simply a parameter (albeit one directly related to intervals measured by a clock) by which the evolution of physical systems are gauged. This parameter role also does not inform us about the nature of time.

\section{Time in the theories of relativity}

Albert Einstein publicly introduced his Special and General Theories of Relativity in the years 1905 and 1915 respectively. ${ }^{15}$ They are two of the most empirically corroborated theories in the whole of science. Relativity has informed us more about the nature of time than any other theory in the history of science and has still more to reveal. Time plays a special role in the Theories of Relativity, over and above being just a physical parameter, as time is also an intrinsic coordinate in these theories. ${ }^{16}$ In other words, time itself is part of what Relativity describes. Also in Relativity, one cannot hypothesise about the nature of time in total isolation from its relation to space. Time and space are not independent of each other, as we shall see below. This is a consequence of the speed of light in vacuum being a universal constant and thereby having the same value for all observers independent of their motion. If observers in different states of motion always find this same value then their measurements of space and time must differ. Further, Relativity requires that the speed of light in vacuum is the fastest speed for transfer of energy or the transmission of information. This 'ultimate speed limit' ensures the maintenance of causality, that is, the preservation of the temporal order of events. ${ }^{17}$ If this limitation did not apply then circumstances could occur where cause and effect in the observable macroscopic world get reversed resulting in logical contradictions!

The Special Theory of Relativity shows that there is no absolute simultaneity between spatially distant events or with objects having different velocities. ${ }^{18}$ Whether two or more events are simultaneous is not fixed by nature but is relative to the circumstances of different observers. This means that the present

$14=0.000000000000000012$ of a second.

15 Pais 1982: 239.

16 Kroes 1985: 77-82.

17 Goldberg 1984: 116.

18 Wald 1992: 14. 
moment in time is relative to different observers (or in physics parlance, relative to different frames of reference) and consequently that there is no universal present moment, that is, my 'now' is not necessarily your 'now'. ${ }^{19}$

Discrepancies in time for different observers (that is, in different frames of reference) would only become obvious when the relative speed of one observer to another is more than half the speed of light in vacuum. However, even at much slower speeds, these differences are measurable. This phenomenon is called 'time dilation' and is usually depicted in a hypothetical scenario with two observers, one of whom goes off on a round trip through outer space in an advanced spacecraft at a speed close to the speed of light. On return to the Earth, the two observers compare their clocks to find the travelling clock reads much less than the stay-at-home clock (and the travelling observer is correspondingly younger than the stay-at-home one).$^{20}$ In spite of time dilation being counter to common sense, tests conducted using elementary particles on one hand, and airborne atomic clocks on the other, have experimentally verified time dilation to an amazing degree of accuracy. ${ }^{21}$

The General Theory of Relativity is a theory about space-time, which is the relativistic union of space plus time. ${ }^{22}$ Space-time may be described as the flexible four-dimensional 'fabric' of the universe. We are all familiar (at least from primary school mathematics) of the three-dimensional geometry of space. General Relativity extends basic geometrical notions by explaining gravity in terms of the four-dimensional geometry of space-time. Gravity is a natural consequence of this four-dimensional geometry. General Relativity also shows that gravitation affects time. It turns out that time intervals between events are not only dependent on relative motion but also on the presence of a gravitational field and its intensity. ${ }^{23}$ This leads to a gravitational version of the time dilation effect, which is also experimentally well supported. ${ }^{24}$ The closer that a clock is to the source of a gravitational field, the shorter will be the time intervals measured by the clock. Since the time differences between different observers on the Earth are so miniscule, we would never notice them. However, they still have to be taken into account in some applications. A hand-held receiver linked to the satellite-based Global Positioning System (GPS), for example, can determine one's position on the Earth to within a few metres. The GPS incorporates corrections due to Relativity effects without which the error in any GPS navigational fix would progressively accumulate. ${ }^{25}$

19 Penrose 1989: 392.

20 Wald 1992: 24-26.

21 Greene 2004: 50.

22 Wald 1992: 34.

23 Angel 1980: 205.

24 Chou et al. 2010.

25 Pascual-Sánchez 2007: 263. 


\section{Time in quantum mechanics}

Elements of Quantum Mechanics were conceived by a number of physicists in the 1920s, especially by Louis de Broglie, Albert Einstein, Erwin Schrödinger, Werner Heisenberg, Neils Bohr and Max Born. ${ }^{26}$ It is another of the most empirically corroborated theories in the whole of science. Quantum Mechanics describes microscopic phenomena, that is, phenomena in the realm of the atom and sub-atomic (quantum) particles. Although Quantum Mechanics has a number of very bizarre consequences, it does not effectively alter the classical notion of time in depicting the states of quantum level physical systems. Quantum Mechanics describes a physical system by means of Schrödinger's Equation, which allows earlier and later states of the system to be calculated. ${ }^{27}$ Time as it appears in Schrödinger's Equation is merely a parameter determined by something external to the physical system being studied, for example, as measured by a clock. Despite this, Quantum Mechanics may provide some important clues for an investigation of time and of the ontological status of events at the microscopic level (that is, whether microscopic events which have already occurred or events which are yet to occur can be said to be real in some sense).

A highly significant result that is inferred from Quantum Mechanics is that the universe is inherently non-local at the level of quantum interactions. ${ }^{28}$ Non-locality refers to the existence of some form of action-at-a-distance, indicating that there are influences which act with speeds faster than the speed of light in vacuum. This conclusion is based on a large number of experimental results that show correlations between spatially distant quantum events. Suppose we have two quantum particles, for example, which are initially produced from a single physical process. Quantum Mechanics tells us that the physical states of these two particles will not be independent. (The technical term for this is that the particles are entangled). If we move one particle a large distance away and then make a measurement of a particular characteristic of the close particle, the corresponding characteristic of the distant particle changes instantaneously. ${ }^{29}$

However, it has been shown that 'quantum non-locality' effects cannot be used to send any form of communication faster than the speed of light in vacuum, ${ }^{30}$ which has avoided a direct clash with Relativity. Since Relativity actually forbids causal propagation that is faster than light, the existence of

26 Enge et al. 1972: 161

27 Kroes 1985: 84.

28 Riggs 2009: 104-105.

29 Rae 2004: 57.

30 Riggs 2009: 114-115. 
non-local effects may eventually demand that the accepted account of relative simultaneity be modified. Indeed, quantum non-locality may turn out to have strong ramifications for our understanding of time.

\section{Philosophical concepts of time}

Philosophical theories cannot be empirically tested. The dividing zone between philosophical and scientific theories has shifted over the millennia. In ancient times, many topics which we would today consider to be science could only be the subject of philosophical debate. A classic example is the question of whether the ultimate nature of matter was atomic, as asked by the ancient Greeks. This question was settled by experiment early in the twentieth century. In recent years, a few other issues that were traditionally considered metaphysical (such as questions relating to the realist view of science) have been the subject of indirect tests through experiments on quantum mechanical systems. Whilst it is clear that physics has uncovered some characteristics of time, methods for directly testing theories of time have not yet been devised. While it is conceivable that experiments might be developed which would discriminate for or against a particular theory of time, it is the case that theories about the nature of time remain in the philosophical domain.

There are many philosophical questions asked in relation to time. Most frequently asked questions about the nature of time include: Does time exist? Are past and future as real as the present? Does time 'flow'? Does time have a 'direction'? The majority of philosophers are of the view that time does exist. It is just that they do not agree on what time is! $!^{31}$ Such questions as these assume a familiarity with the terminology used. If time does flow (in some sense) then one would expect it to be directed. In other words, the flow of time ought to 'progress' strictly in one orientation of time. The direction of time is a stronger concept than time orientation as the latter concept is neutral in regard to 'which way' in time. The graphical analogy for the direction of time is a directed straight line, that is, a line that includes an arrow which consistently points along only one of the orientations of time. There are a number of theories postulated in the philosophy of time, each of which provides different answers to questions about time. We shall now canvass the most popular philosophical theories of time and see what answers they offer. 


\section{Causal theories of time}

Causal Theories of Time have had much attention in the philosophy of time literature. These are members of the set of relational theories of time. In relational theories, time is postulated to be purely relative to events, that is, time does not exist in itself. Instead, events are considered fundamental and what we perceive as time is constituted by the existence of particular relations between events. ${ }^{32}$ Relations between events or objects (in the philosophical sense) express real characteristics or connections between the events or objects, for example, for the relation of 'being taller than' to hold between two people, one of them must have a longer body length than the other.

We shall deal with the general thrust of these causal theories. They concern causal relations between events, that is, the relationships of causes to effects. It is asserted in the Causal Theories that causal relations are more basic than temporal relations, where temporal relations concern whether one event occurs before another in time. ${ }^{33}$ If we have two events where one event causes the other event, the causal order of the events is determined by which of these events is the cause. This determination is logically independent of the temporal order of the events. Causal order is defined as the order where the event that is the cause is first, or primary, and the event that is caused (the effect) is second. Causal relations are asymmetric and transitive such that if $\mathrm{A}$ is a cause of $\mathrm{B}$ and $B$ is a cause of $C$ then $A$ is a cause of $C$, but not vice-versa. In this view, causes in all circumstances must temporally precede their effects. The temporal order of events is thereby derivative from their causal order. Causal Theories of Time suffer from at least one major flaw. In all attempts to show the reduction of temporal relations to causal relations, implicit appeal has been made to temporal notions, which then undermined the whole endeavour of attempting to make this reduction. ${ }^{34}$

\section{Statistical theories of time}

Statistical Theories of Time are principally about the origin of the direction of time. The original Statistical Theory of Time held that our concept of time is dependent on the observed fact that physical systems when left alone will tend to become more disorganised at later times. This is quantified in terms of the entropy of a closed physical system. Entropy is a measure of disorder and

32 Bardon 2013: 14.

33 Sklar 1977: 319.

34 Whitrow 1980: 326; Kroes 1985: 19. 
refers to the physical system as a whole rather than its individual constituents. Your home garbage has, for example, a lower entropy when stacked in your rubbish bin (less disorder) than when your neighbour's dog has spread the bin contents all over your home's front yard. Indeed, throughout our lives we see all sorts of natural processes that result in more disorder, such as biological decay and ageing.

The entropy of a closed physical system is postulated never to decrease. (In physics, this is a statement of the Second Law of Thermodynamics which, although being a law of physics, is not one of the fundamental laws referred to above.) A closed physical system, regardless of its size, is necessary in order to rigorously define entropy. However, it became clear that because the original Statistical Theory of Time dealt with the average variation of entropy in a closed system, it could not rule out periodic decreases in entropy, ${ }^{35}$ and therefore is unable to unambiguously define an objective direction of time. Other attempts to define an objective time direction with respect to entropy increase have also failed. ${ }^{36}$ This failure has undermined attempts to show that entropy-increasing activities in the human brain are responsible for generating our sensations of time (more about temporal sensations appears below).

Another Statistical Theory of Time appeared when the Noble Prize-winning physicist Richard Feynman put forward an account of the interactions of elementary particles after the discovery of anti-matter. An anti-matter particle has the same mass as the corresponding particle of ordinary matter but an opposite electric charge. An anti-electron (called a positron), for example, has a positive electric charge whereas an electron has a negative electric charge, but both have identical mass. In Feynman's account, anti-matter particles are considered to be matter particles moving 'backwards' in time (that is, along the orientation of time called earlier). This led to the idea that there might not be a unique time direction at microscopic scales. The familiar macroscopic time direction was then theorised to be a statistical effect due to the predominance of matter over anti-matter in the universe.

This version of the Statistical Theory of Time has some odd consequences. In particular, if our macroscopic time direction depends on there being only extremely small numbers of anti-particles, then time direction would disappear in any spatial region that contained a large amount of anti-matter! Feynman's account lacks evidence and is not taken seriously by most physicists. Consequently, this Statistical Theory of Time is not considered viable.

35 Whitrow 1980: 331-332; Bardon 2013: 14.

36 Price 2011: 284-285. 


\section{Realist theories of time}

The realist perspective of time is that temporal relations between events are more basic than other relations and that time has an objective existence beyond mere temporal relations. There are essentially two realist theories of time. These two theories are known by several names in the literature of philosophy of time. The most commonly used names are the A-Theory (or Dynamic Time) and the B-Theory (or Block Time). The ontologies of these realist theories (that is, what they postulate to exist) are distinct and incompatible.

\section{The A-Theory of time}

The main features of the A-Theory may be listed as follows: ${ }^{37}$

- The primary relations between events are the tensed temporal relations of past; present; and future.

- The flow of time (also called passage of time or temporal becoming) by which the present moment 'moves' from past to future, entails an objective coming into being of events.

- Time has an intrinsic direction from past to future.

- Changes are only understandable in terms of tensed temporal relations.

Central to the A-Theory is the notion of an objective present moment (the 'now'), which 'moves' from past to future and is perceived as the flow of time. The present moment is a special point of time known from conscious experience that separates the closed past from the open future and is characterised by the process of temporal becoming. Temporal becoming changes the status of an event from unactualised to actualised (that is, the process by which an event comes into existence). Therefore, the past is determined and the future is undetermined. Consequently, according to the A-Theory, singular statements can be made about past events, but statements about future ones can only be general in their form.

If temporal becoming is a change in the ontological status of events from an undetermined to a determined state, then the question to be asked is determined for whom? A standard response is that an event is determined for us at this particular moment. However, by answering in this way, we merely have

37 Gale 1968: 77; Price 1996: 12-13; Dainton 2010: 10-12. 
defined the present moment with respect to itself. ${ }^{38}$ Such a subjective account is completely unsatisfactory. Indeed, no attempt to objectively define the present moment has succeeded.

In the A-Theory, the universe is intrinsically irreversible as a consequence of temporal becoming. Therefore, the observed temporal asymmetry of events arises from time's dynamic nature. The direction in which irreversible processes occur (for example, cream becoming butter) gives the direction of time.

One version of the A-Theory that has received much attention in recent years is called Presentism. This is the view that only objects and experiences in the present actually exist (where 'present' means temporally present as distinct from spatially present)..$^{39}$ According to Presentism, anything that lacks the property of being present is unreal. Therefore, Presentism asserts that the past and future as such do not exist. Although Presentism remains popular amongst a minority of philosophers, there are robust arguments in the philosophical literature against it. In particular, the case that Presentism conflicts with the Special Theory of Relativity is well established. ${ }^{40}$ These arguments (some invoking Relativity and others based on purely logical grounds) cast serious doubt on the truth of Presentism.

\section{The B-Theory of Time}

The main features of the B-Theory may be listed as follows: ${ }^{41}$

- The primary relations between events are the tenseless temporal relations of earlier than and later than.

- There is no flow of time or objective coming into being of events.

- There is no objective present moment.

- All events are equally real.

- Changes do not require tensed temporal relations.

- Temporal asymmetry is due to the boundary conditions that apply to physical processes.

In the B-Theory, what appears to be past, present or future is purely subjective and the 'now' is observer dependent. The tensed relations of the A-Theory are taken as not being objective but instead relative to particular events in much

38 Whitrow 1980: 349.

39 Dowden 2013.

40 See, for example, Saunders 2002; Wüthrich 2013.

41 Gale 1968: 70; Price 1996: 12-13; Dainton 2010: 10-12. 
the same way as spatial tenses (for example, 'here' and 'there') are relative terms. Singular propositions about events are (tenselessly) true or false, although one might not be aware of what the truth value of a particular proposition is.

The B-Theory can accommodate the fact that the world appears to be asymmetric in time by noting that there are (de facto) irreversible processes which result from physical boundary conditions. Therefore, temporal asymmetry is not intrinsic to time in the B-Theory but arises from these boundary conditions. We do not see mixed coffee and milk spontaneously separate in a cup, for example, because of the boundary conditions imposed by putting these liquids into the cup (pouring one liquid into the other, limiting the space in which the liquids can spread, and so on). These conditions ensure that the probability of the coffee and milk spontaneously un-mixing is so small that it would take longer than the current age of the universe for such an event to occur.

If the B-Theory of time is correct, it would explain much about our universe and why the laws of physics take the forms they do (being time-reversal invariant). Yet, the B-Theory is not unproblematic. A major failing of the B-Theory is that it does not offer a sufficient explanation of the common feeling that there is a flow of time from the past to the future, merely ascribing this feeling to being a psychological phenomenon. ${ }^{42}$

\section{Ultra deep time}

If deep time dates back to the formation of the Earth, then it follows that 'ultra deep time' dates back to the origin of the universe. Precise astronomical measurements in the 1920s (and validated throughout the rest of the twentieth century) have shown that the universe is expanding, that is, the galaxies are getting further apart at later and later times. ${ }^{43}$ Therefore, if we (theoretically) retrace the motion of the galaxies far enough back in time, then we reach a time in the history of the universe where all the galaxies would be at the same point. This was the origin of the whole universe. According to the currently accepted scientific cosmological view, the universe began with a tremendous burst of energy approximately 13.8 billion years ago, which is called the Big Bang. ${ }^{44}$ This was not an 'explosion' into a pre-existing empty space, for the Big Bang constituted the creation of space and time. If this is correct, then time itself

42 Price 1996: 12-14.

43 Tegmark 2014: 45-46.

44 Singh 2004, chap. 5; Tegmark 2014: 44-46. 
started at the Big Bang! Surprisingly perhaps, Saint Augustine eloquently and succinctly expressed the gist of this idea when he wrote 'verily the world was made with time, not in time' ${ }^{45}$

Current astrophysical evidence indicates that the rate of the universe's expansion is actually accelerating. ${ }^{46}$ Such acceleration has a number of serious implications, including whether the expansion provides an objective basis for temporal asymmetry, whether time will have an ending (or will continue indefinitely), and indeed, whether a physical definition of time will hold in a global sense.

There are theoretical alternatives to the standard cosmological view in which there is 'something' prior to the Big Bang (or no Big Bang at all). These alternative theories postulate either an eternally existing universe or that new, whole universes are created in a never-ending cosmic process ${ }^{47}$ However, the alternatives are not faring well as recent theoretical and observational findings support the proposition that the Big Bang was a physical boundary of time. ${ }^{48}$ This physical boundary is indicated by calculations showing that the paths of objects in space-time cannot be continued indefinitely to earlier times and therefore cease (at the Big Bang). ${ }^{49}$ The Big Bang remains the best supported theory of physical cosmology.

We should also note that the study of the very earliest moments of the universe (at a time of less than $10^{-43}$ of a second after the Big Bang ${ }^{50}$ ) is hampered by the problem that our current theories fail when applied to this early era. ${ }^{51}$ In order to deal with physical systems in the most extreme conditions (such as obtained in the very early universe), we need a theory that combines General Relativity and Quantum Mechanics. This is a theoretical unification called Quantum Gravity and, despite decades of effort, is still to be achieved. The theory of Quantum Gravity is expected to provide some important insights about time, although exactly what these will be await the arrival of the theory!

There is another sense of ultra deep time which concerns the ultimate nature of time at the smallest physical scale for space, time and energy. This is known as the Planck scale. The physical constants of nature set the Planck scale at about $10^{-35}$ metre. ${ }^{52}$ At this level, a principal question is whether the structure of time is discrete or continuous. There are persuasive arguments to the effect that physical space-time has a granular constitution that would only become evident

45 Quoted in Whitrow 1980: 33, fn. $\ddagger$ (italics added).

46 Tegmark 2014: 77.

47 Tegmark 2014: 151-152.

48 Moss et al. 2011; Grossman 2012.

49 Guth 2007: 6821-6824.

$50=0.0000000000000000000000000000000000000000001$ of a second

51 Adler 2010: 931.

$52=0.00000000000000000000000000000000001$ of a metre. 
at extremely minute distances. ${ }^{53}$ If so, this would also imply that time is discrete and only appears to be continuous at levels much larger than the Planck scale. A discrete structure of time would have significant consequences for physical theories in general, as most assume time to be continuous at all scales.

There are also experimental results that may influence our understanding of time, which have been noticed in some kinds of sub-atomic particle interactions. These results, gained by examining data from billions of particle collisions, strongly suggest that a form of temporal anisotropy exists. ${ }^{54}$ Temporal anisotropy is a structural difference between the two orientations of time. If temporal anisotropy does exist, it could be used to objectively and consistently distinguish between the two orientations of time. Additional experiments are needed to study the relevant particle interactions and gather more data before temporal anisotropy can be considered to have been firmly established. Clearly though, if the proposals about time possessing the properties of discreteness and anisotropy can be supported by sizeable and robust amounts of empirical evidence, then the implications for the deep structure of time are profound.

\section{Prospects for a more complete understanding of time}

Of the philosophical theories canvassed, it is the B-Theory that fits best with Relativity, which makes the B-Theory quite attractive. In this context, spacetime is interpreted as being the totality of events, also called the 'Block Universe'. All events in the Block Universe have the same ontological status, that is, are equally real regardless of when they occur (as also postulated in the B-Theory). We also have acknowledged that the B-Theory does not properly account for the common feeling that there is a flow of time. In order to address this failure, the B-Theory needs to offer an explanation of this feeling that is comprehensible in terms of objective features of the universe. It should not be surprising then, that attempts to find improved philosophical explanations for the feeling that time flows that are consistent with the B-Theory constitute an area of ongoing philosophical research. ${ }^{55}$

It was also previously noted that the fundamental laws of physics do not specify an objective 'moving' present moment as implied by human consciousness. This has led some philosophers to allege that physics has not explained a basic

53 Greene 2004: 490-491; Adler 2010.

54 Schwarzschild 2012: 16.

55 See, for example, Riggs 2012; Prosser 2013; Deng 2013. 
feature of reality and, consequently, to claim that physics is incomplete in its description of time. In order to address this claim, answers would need to be sought to the following questions:

- What is the relevant aspect that might be missing in the physical account of time?

- How could the missing aspect be discovered?

- By what mechanism would the missing aspect bring about human temporal experiences?

Finding answers to these questions and, more generally, gaining a better understanding of the nature of time may require a cross-disciplinary approach. Interestingly, the amount of research in the fields of experimental psychology and cognitive science into time perception has increased markedly over the first decade of this century. ${ }^{56}$ Much of this research has centred on judgements about temporal intervals and how the brain might process such intervals. ${ }^{57}$ However, the neurophysiological basis of the human experience of time is still unknown. ${ }^{58}$ What is almost totally lacking in the cognitive experimental arena are rigorous tests into the conscious feeling of the flow of time. Such tests might prove exceedingly valuable for they may assist in discovering whether this feeling is purely mind dependent (as postulated in the B-Theory) or not. ${ }^{59}$

Given the rapid and in-depth development of physical science that has occurred since the early twentieth century, it is likely that further advances in physics will result in discoveries of new aspects of time. In particular, the theory of Quantum Gravity should provide novel physical insights into time. However, improving our knowledge about time might be best achieved by integrating philosophical ideas with those of physics and cognitive science. At the very least, an integrated approach should assist in identifying gaps in our understanding of time. It is an encouraging development for the study of time that philosophers and physicists are starting to engage in common dialogues on issues of mutual interest. The integration of the philosophical and the physical will also have the potential to solve some of the conceptual problems of modern physics that remain outstanding (such as quantum non-locality). The cosmologist Lee Smolin offered the following commentary about the relation of time to our understanding of the physical universe:

$[\mathrm{T}]$ he extent to which we bring laws of physics inside of time is the extent to which we make them amenable to rational understanding. Time is then

56 Ivry and Schlerf 2008: 273; Eagleman and Pariyadath 2009: 1841.

57 See Grondin 2010, for a review.

58 Wittmann et al. 2010: 3110.

59 Some proposed tests are outlined in Riggs 2012. 
the key for the aspiration to construct a theory of the whole universe ... Time is thus the most central and most difficult problem we must face as we attempt to construct a theory of a whole universe. ${ }^{60}$

Research into the nature of time has an exciting future and one which holds the potential for finding solutions to some of the most baffling questions asked in the history of human thought.

\section{Bibliography}

Adler, Ronald J 2010, 'Six easy roads to the Planck Scale', American Journal of Physics 78(9): 925-932.

Angel, Roger B 1980, Relativity: The Theory and its Philosophy, Pergamon, Oxford.

Aveni, Anthony 2000, Empires of Time: Calendars, Clocks and Cultures, Tauris Parke, London.

Bardon, Adrian 2013, A Brief History of the Philosophy of Time, Oxford University Press, Oxford.

Chou, CW, DB Hume, T Rosenband and DJ Wineland 2010, 'Optical clocks and relativity', Science 329(5999): 1630-1633.

Dainton, Barry 2010, Time and Space, second edition, Acumen, Durham, UK.

Denbign, Kenneth 1981, Three Concepts of Time, Springer, Berlin.

Deng, Natalja 2013, 'On explaining why time seem to pass', Southern Journal of Philosophy 51: 367-382.

Douglas, Kirsty 2010, Pictures of Time Beneath: Science, Heritage and the Uses of the Deep Past, CSIRO Publishing, Canberra.

Dowden, Bradley 2013, 'Time', in The Internet Encyclopedia of Philosophy (ISSN 2161-0002), www.iep.utm.edu.

Eagleman, David M and Vani Pariyadath 2009, 'Is subjective duration a signature of coding efficiency?', Philosophical Transactions of the Royal Society B 364: 1841-1851.

Elton, Lewis RB and Harry Messel 1978, Time and Man, Pergamon, Oxford.

60 Smolin 1997: 262 
Long History, Deep Time

Enge, Harald A, M Russell Wehr and James Austin Richards 1972, Introduction to Atomic Physics, Addison-Wesley, Reading, MA.

Fraser, Julius Thomas 1987, Time, the Familiar Stranger, Tempus Books, Redmond.

Gale, Richard M (ed.) 1968, The Philosophy of Time: A Collection of Essays, Macmillan, London.

Goldberg, Stanley 1984, Understanding Relativity: Origin and Impact of a Scientific Revolution, Clarendon Press, Oxford.

Gould, Stephen J 1987, Time's Arrow, Time's Cycle: Myth and Metaphor in the Discovery of Geological Time, Harvard University Press, Cambridge, MA.

Greene, Brian 2004, The Fabric of the Cosmos: Space, Time and the Texture of Reality, Penguin, London.

Grondin, Simon 2010, 'Timing and time perception: A review of recent behavioral and neuroscience findings and theoretical directions', Attention, Perception, \& Psychophysics 72: 561-582.

Grossman, Lisa 2012, 'Death of the eternal cosmos', New Scientist (14 January 2012): 6-7.

Guth, Alan 2007, 'Eternal inflation and its implications', Journal of Physics A 40: $6811-6826$.

Ivry, Richard B and John E Schlerf 2008, 'Dedicated and intrinsic models of time perception', Trends in Cognitive Sciences 12: 273-280.

Kroes, Peter 1985, Time: Its Structure and Role in Physical Theories, Reidel, Dordrecht.

Moss, Adam, Douglas Scott and James P Zibin 2011, 'No evidence for anomalously low variance circles on the sky', Journal of Cosmology and Astroparticle Physics 4: 1-7.

Pais, Abraham 1982, Subtle is the Lord...: The Science and the Life of Albert Einstein, Oxford University Press, Oxford.

Park, David 1980, The Image of Eternity: Roots of Time in the Physical World, University of Massachusetts Press, Amherst, MA.

Pascual-Sánchez, J-Fernando 2007, 'Introducing relativity in global navigation satellite systems', Annalen der Physik 16: 258-273. 
Penrose, Roger 1989, The Emperor's New Mind: Concerning Computers, Minds, and the Laws of Physics, Vintage Press, London.

Phys.org News 12 May 2010, '12 attoseconds is the world record for shortest controllable time', Phys.org (online), phys.org/news 192909576.html.

Price, Huw 1996, Time's Arrow and Archimedes' Point: New Directions for the Physics of Time, Oxford University Press, Oxford.

Price, Huw 2011, 'The flow of time', in Craig Callender (ed.), The Oxford Handbook of Philosophy of Time, Oxford University Press, Oxford.

Prosser, Simon 2013, 'Passage and perception', Nô̂s 47: 69-84.

Rae, Alastair IM 2004, Quantum Mechanics: Illusion or Reality?, second edition, Cambridge University Press, Cambridge.

Reichenbach, Hans 1956, The Direction of Time (ed. Maria Reichenbach), University of California Press, Berkeley and Los Angeles.

Riggs, Peter J 2009, Quantum Causality: Conceptual Issues in the Causal Theory of Quantum Mechanics, Springer, Dordrecht.

Riggs, Peter J 2012, 'What do we feel when we "feel" time "passing"?', Journal of Consciousness Exploration \& Research 3: 1064-1073.

Rovelli, Carlo 1995, 'Analysis of the distinct meanings of the notion of «Time», in different physical theories', Il Nuovo Cimento B 110: 81-93.

Saunders, Simon 2002, 'How relativity contradicts presentism', in Craig Callender (ed.), Time, Reality and Experience, Cambridge University Press, Cambridge, 277-292.

Schwarzschild, Bertram M 2012, 'Time-reversal asymmetry in particle physics has finally been clearly seen', Physics Today 65(11): 16-18.

Shryock, Andrew and Daniel Lord Smail 2011, Deep History: The Architecture of Past and Present, University of California Press, Los Angeles.

Singh, Simon 2004, Big Bang: The Most Important Scientific Discovery of All Time and Why You Need To Know About It, Fourth Estate, London.

Sklar, Lawrence 1977, Space, Time and Spacetime, University of California Press, Berkeley and Los Angeles.

Smolin, Lee 1997, Life of the Cosmos, Phoenix, London.

Tegmark, Max 2014, Our Mathematical Universe, Knopf, New York. 
Long History, Deep Time

Wald, Robert M 1992, Space, Time, and Gravity, second edition, Chicago University Press, Chicago.

Wittmann, Marc, Alan N Simmons, Jennifer L Arona and Martin P Paulus 2010, 'Accumulation of neural activity in the posterior insula encodes the passage of time', Neuropsychologia 48(10): 3110-3120.

Whitrow, Gerald J 1980, The Natural Philosophy of Time, second edition, Clarendon Press, Oxford.

Wüthrich, Christian 2013, 'The fate of presentism in modern physics', in Roberto Ciunti, Kristie Miller and Giuliano Torrengo (eds), New Papers on the Present: Focus on Presentism, Philosophia Verlag, Munich, 91-131. 
This text is taken from Long History, Deep Time: Deepening Histories of Place, edited by Ann McGrath and Mary Anne Jebb, published 2015 by ANU Press, The Australian National University, Canberra, Australia. 